\title{
ZU SWEET'S OLDEST ENGLISH TEXTS. I.
}

Im Beiblatt zur Anglia VI ${ }^{6}$ (October 1895) pg. 164 tadelt Dieter in seiner besprechung von Hall's Anglo-Saxon Dictionary, dass das von Sweet Oldest English Texts pg. $463^{\text {a }}$ angesetzte Adjektiv staefod 'striped' keine aufnahme gefunden habe. Ich denke, ein näherer blick auf die Glossen, auf welche sich Sweet's ansatz stützt, wird uns lehren, dass Hall mit recht die aufnahme verweigerte. Das Erfurter Glossar hat nach Goetz's Corpus Gloss. Lat. V 385, 12 perstromata ornamenta staefadbrum, das Epinaler p. o. stefad brum, das Corpus Christi Glossar aber nach Hessels sorgfältiger ausgabe p. 357 p. $o$. steba. Es entging Hessels nicht, dass alle diese glossen eine verdorbene wiedergabe von peristromata ornamenta stibadiorum sind und zum überflusse hatte, wie er angiebt, schon Loewe in seinem Prodromus pg. 347 darauf hingewiesen. Stibadium ist natürlich eine neutrale bildung vom griechischen $\sigma \tau \iota \beta \dot{\alpha} \varsigma$, das in der schreibung $\sigma \tau o \iota \beta \dot{\alpha}_{S}$ im Markusevangelium erscheint

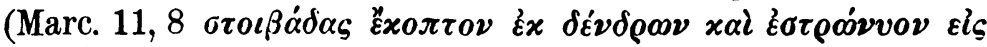

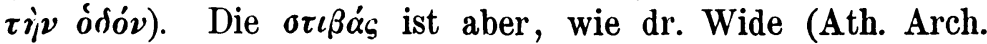
Mitt. XIX, 272) bemerkt, eigentlich dasselbe wie streu oder ein einfaches lager von blättern, schilf u. dgl. (cf. Xenoph. Hell. VII, 1, 16, Polyb. V, 48, 4), woraus im laufe der zeit ein gepolsterter ruhesitz geworden ist, in welchem sinne stibadium an unserer stelle steht. Die schreibung des griechischen wortes im Marcusevangelium zeigt übrigens deutlich, wie die verderbnis der obgenannten Glossare entstehen konnte. Lateinisch stibadium geht doch wohl auf eine griechische nebenform $\sigma \tau \iota \beta \dot{\alpha} \delta \iota 0 \nu$

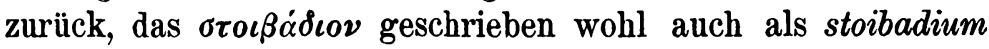
(stoebadium) im Lateinischen figurierte.

Wurde dann nach der bekannten manier $b$ mit $u$ vertauscht und dieses, wie so oft in diesen glossen geschieht, 
durch $f^{\prime}$ ersetzt, so entstand eine form stoefadiorum, der die obigen verderbnisse ihren ursprung verdanken, die Sweet als angelsächsisch stafod 'striped' und brūn 'cloth' ansah.

Auf ähnliche weise ist Sweet OET pg. $644 \mathrm{~b}$ auch zu einem ansatz $c \bar{o} c$ 'cook' gekommen. Das Erfurter Glossar (C. Gl. L. V 354, 28) hat culinia coacas; das Corpus Gl. (C. 953): culinia cocas und dies erscheint WW 366, 28 als culini cocas wieder. Diese form muss Sweet im auge gehabt haben, als er sein $c \overline{o c}$ 'cook' ansetzte, Jedenfalls hat er dies culini als plural von einem anzunehmenden *culinus 'küchenmann' aufgefasst und in culinia eine verderbnis von culini gesehen. Anders weiss ich mir wenigstens seinen ideengang nicht zu erklären. Aber jenes coacas des Erf. Gl. ist sicher aus einem ursprünglichen cloacas entstanden und culinia ist entweder verstümmlung oder abkürzung von sterculinia. Man vergleiche die folgenden glossen: C. Gl. L. IV 326, 34 culna latrina secessum

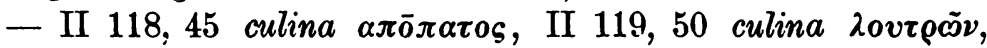

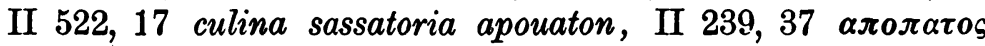

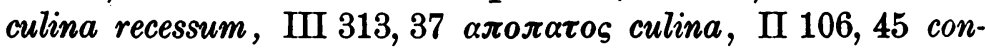

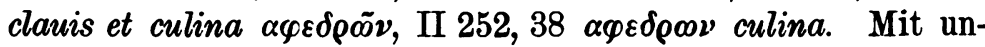
recht hat daher Kolkwitz (Anglia XVII 4 pg. 455) dies cōcas als eine gesicherte angelsächsische form für seine lautstudie verwandt.

Vollständig aus der luft gegriffen ist der ansatz eorðcrypel 'paralytic' (OET pg. $574 \mathrm{a}$ ). Denn was im Corpus Glossar (A 706) steht

\section{applare eorscripel}

berechtigt nicht im entferntesten zu einer solchen annahme, wiewohl Hall das wort seinem Dictionary einverleibt hat. Applare ist wahrscheinlich verderbnis von auriculare (sc. instrumentum) und eor-scripel ist = ear-scripel 'ohren-kratzer' vgl. C. Gl. L. II 26, 42 aurisclarium (d. h. auriscalpium) øгo-

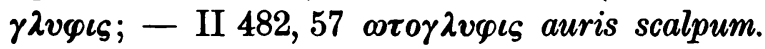

Nicht viel besser steht es mit den ansätzen nëwe-sẽoða 'pit of the stomach' und rāegc-rèose 'spinal muscles' (OET pg. 619 a). Ersteres gründet sich auf die Epinaler Glosse (C. Gl. tatum.

${ }^{1}$ vgl. Corpus Gl. D 137 defectum . deportatum = deuectum . depor- 
L V 365, 43) ilium neuñ seada; letzteres auf die Erfurter 3 (C. Gl. L. II 584, 35 inguen lesĩa hregresi. Von dem lateinischen Lemma schon ist ersichtlich, wie willkürlich Sweet's erklärung beider worte ist. Doch sehen wir uns die überlieferung einmal genauer an. Das Epinal Glossar hat, wie erwähnt

ilium neũ̃ seada,

das Erfurter 1 (C. Gl. L. V, 365, 43)

ilium naensida

und das Erfurter 3 (C. Gl. L. II 582, 15)

ilium neisñ naensõod.

Vergleichen wir WW 419,9 ilibus smaelpearmum

159, 36 ilia smaelepearmas

26, 6 ilia midhridir

517, 14 ilia innepas,

so dürfte es wohl nicht zu gewagt erscheinen, in neisn ein eisn d. h. iesn, das nach Wülkers falscher lesung WW 20, 24 als lesenexta erscheint, aber nach Hessels sorgfältiger ausgabe (A 439) (exta) iesen ist und WW 393, 11 in der form isen 1 (exta) auftritt. Mit diesem iesen steht jedenfalls das in den Northumbrischen Evangelien so oft vorkommende esne im zusammenhang, das bald zur übersetzung von servus, bald von adolescens, juvenis dient und wohl ursprünglich 'virilia' 'pubes' bedeutet, daher denn auch esnelice in der bedeutung von viriliter vorkommt. Dasselbe wort steckt wohl auch WW 77, 40 in iunges (adolescens), wo iungês = iung esn sicher am platze ist. Was nun neũ̃ seada naensida, naensõõd anbelangt, so haben wir wohl von der überlieferung des Erfurt 1 auszugehen, um zum verständnis des wortes zu gelangen. $n$ und $r$ werden in diesen glossaren unzählige male verwechselt, es liegt daher nahe, naersida d. h. near- (neor-) sida zu lesen und demgemäss ist neuñ seada wohl = neru seada und naen sõod = naer seoda d. h. near (neor) seoda; neor-sida würde eine bildung sein wie heort-gesida das Aelfric (Lev. 3,3) zur wiedergabe von vitalia verwendet, oder sulhgesidu im Gerefa 17 ('acker-ge-

1 Den dativ plur. davon haben wir wohl in isernum (=isennum) intestinis Lorica Gl. 71 und in dem von Cockayne Leechd. I pref. LXXII aufgeführten isernum ( $H$ eosenum) za konstatieren. Der volle wortlant bei C. ist turtuosis cum intestinis : gebegdum cam isernum (eosenum). 
schirr'); neor-sida' also wohl 'nieren - gedärme - geschirr' bedeuten; wegen der auffassung von 'niere' vgl. Ahd. Gl. II 374, 37 rien, testiculus niero. Nun zu inguen lesc̃a hregresi: Obwohl als angelsächsisch von Sweet markiert, hat lesca doch in seinem glosasre keine beachtung gefunden, pg. 619 a führt er es aber also iesca an; die lesung lesca ist jedoch die allein richtige und es ist = leosca 'schamleiste, schambug' $=$ mittelenglisch lêske; ein davon gebildetes verb erscheint WW 106, 31 spadatus, eunuchizatus belisnod = beliscnod und in der angels. Evangelienübersetzung (c. 1000) Matth. 19, 12 eunuchizati belistnode = beliscnode. Damit vgl. Abraham a Santa Clara (1684) pg. 9: 'Reim dich oder ich Lisz dich'.

Auf ebenso unsicherer grundlage wie die vorigen ruht der ansatz (OET 584 a) grundsopa 'groundsoap (a plant)'. Auch hier hatte Loewe (Prodromus pg. 418) schon das richtige gezeigt. Die überlieferung, auf die Sweet seinen ansatz stützt, ist die folgende: Das Erfurter 1 (C. Gl. L. V 355, 24) hat

cartilago gg. grund suopa,

das Erfurter 2 (C. Gl. L. V 274, 35):

cartilago grurzapa dicitur rusticae

und das Corpus Glossar C. 186 :

\section{cartilago grundsopa.}

In zweien der glossare haben wir eine ausdrückliche angabe über die nationalität des wortes grundsopa, nach der einen ist es griechisch, denn das bedeutet $g g$., nach der andern ist es vulgär-lateinisch. Beides lässt sich vereinigen, wenn die ursprüngliche lesung war:

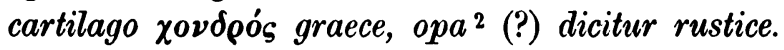

Was hregresi anbelangt, könnte das nicht hegpresi, entsprechend althochdeutschem heyadrosi sein? Wurde dies *hegpresi als hegthresi geschrieben, so war verderbnis in hegtresi, hegcresi sehr leicht, woraus dann unser hregresi werden konnte, indem der schreiber das $r$ der zweiten silbe anticipierte.

1 Es will mich bedünken, dass das reada, das WW 159, 38 als übersetzung von tolia uel porunula erscheint, seada ist und $\mathrm{zu}$ dem vorhergehenden ilia smaele bearmas gehört; $r$ und 8 werden ja auch so oft verwechselt.

2 verdorben aus lapa = lappa? Ich sollte meinen, da wir in diesen glossen auch latinisierte germanische worte treffen, wie z. b. garba WW 24, 2. 413,1. 517, 11 und ganta 28 1, 13, so wäre die möglichkeit vorhanden 'rustice' auch von solchen wörtern zu verstehen. 
Dass der ansatz OET pg. 589 hálstán 'crystal' nicht richtig sein kann, darauf hat Dieter hingewiesen, nachdem bereits Diefenbach in seinem Glossarium die richtige auffassung des wortes unter crustula gegeben hatte. Dieter nimmt als normalform heall-stan, erklärt sich aber ausser stande, nachzuweisen, wie ein wort von solcher zusammensetzung ein gebäck bezeichnen könne. Dies dürfte nicht allzuschwer sein. Zunächst wollen wir bemerken, dass Dieter nicht alle in betracht kommenden stellen angeführt hat. Denn zu WW 364, 36 colliridam healstan

372,17 crustulis healstanum

495, 28 crustule healstanes gehört ohne zweifel auch

216, 5 crustula helsta (d. i. helstã = helstan) uel rinde und 393, 31 et tortan panis and hylstene hlafas.

Hall freilich gewinnt von 216, 5 ein neues wort helsta 'crust' und für 393, 31 statuiert er ein adjektiv hylsten = 'twisted', aber seine aufstellungen haben ja, wie ich zu zeigen hoffe, keinerlei kritischen wert. Wie wir torta aufzufassen haben, geht klärlich hervor aus stellen wie C. Gl. L. III 574, 41 passa i. una torta III 596, 18 unapassa racemus tortus und Num. 11,8,

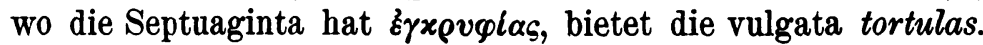
Das heisst doch wohl torta und tortula wurden als ableitungen von torrere und nicht von torquere gefasst und so haben es auch die althochdeutschen glossatoren genommen, denn Ahd. Gl. I 463, 25 wird turtam panis schlankweg mit leip ${ }^{2}$ protes und - I 585, 42 tortura mit leip wiedergegeben; hylstene steht also für hylstaen(e)ne und ist adjektiv von hyl-stan = heal-stan = hal-stan; hal-stan aber steht für al-stan und (h)al- ist dasselbe wort, das uns in den zusammensetzungen aalgewerc ignarium ('Feuer-werk') WW 26, 5 aal-fatu cocula (koch-gefässe) WW 212, 24, auch ol-fata (WW 122, 25) cocula begegnet. Demnach ergiebt sich für (h)alstan als grundbedeutung: Feuer (herd) oder kochstein. Da aber der name des gerätes so oft auf das dadurch hervorgebrachte ding übergeht ${ }^{3}$, so steht

1 Analog dem haben wir WW 527, 26 turtas hlafas.

${ }^{2}$ leip $=$ ags. hlaf wird ja mit $x \hat{\lambda} i \beta \alpha v o s$ in etymologischen zusammenhang gebracht, was zu dem torta gut passen würde.

3 So ist aus frixorium 'röstpfanne' das französische fressure 'geschlinge' geworden, womit man das deutsche 'schlacht-schüssel' ver- 
(h)alstan dann auch für das auf ihm hergestellte gebäck = focacium (suffocacium) = französisch fouasse, und bezeichnet dasselbe, das sonst als herstinghlaf (WW 372, 17), heorðbacen hlaf (WW 153,36) oder cecil (WW 49, 28) erscheint. In der that weist letzteres wort eine ähnliche bedeutungsentwicklung auf, indem es unserm 'kachel' entspricht. Und so erklärt es sich auch, dass Aelfric, wenn er Genes. 18, 6 fac subcinericios panes wiedergiebt, dies durch wirc focan thut, indem er schlankweg dazu das lateinische focus entlehnt und es angelsächsisch umgestaltet.

Ein höchst merkwürdiger ansatz ist pg. 516a tetridit 'tramples'. Die überlieferung im Erfurt 1 (C. Gl.L. V 356, 53) ist: defecit tedridtid;

das Corpus Glossar bietet (D 77):

\section{desicit tetridit.}

Es ist auf den ersten blick klar, dass auch hier wieder der Erfurt die bessere überlieferung bewahrt hat. Denn wir brauchen uns nur zu erinnern, dass $d$ in der form $\delta$ dem $o$ zum verwechseln ähnlich sieht und es springt in die augen, dass wir zu lesen haben

$$
\text { deficit [tempus] teorid tid (= teorið tid). }
$$

Ebenso merkwürdig ist der ansatz (pg. $576 \mathrm{~b}$ )

$$
\text { orc 'demon' }
$$

auf grund der Corpus Glosse (J 152)

\section{inmunes orceas.}

Hall hat den ansatz natürlich für bare münze genommen und seinem wörterbuch einverleibt, aber mir nichts dir nichts anzunehmen, dass der glossator von einem dämon hat sprechen wollen, wenn das lemma gar keinen anlass dazu bietet, ist doch die reine willkür. Es ist klar, dass wir es in orceas mit einem adjektiv $\mathrm{zu}$ thun haben, dessen or- dem in und ceas dem -munes entspricht. Das wort begegnet wieder in genau derselben form und mit genau demselben lemma WW 522, 10

gleiche. So spricht man auch in Philadelphia von 'sauern flecken (kutteln)' als 'peppery pot'. Erhitzte steine aber zum kochen zu verwenden, ist eine alte sitte, die an der Neuenglandküste noch heute zur sommerszeit beim 'oldfashioned clam-bake' geübt wird und die Wüstenaraber backen ihre kuchenartigen flachbrode darauf in derselben primitiven weise wie ihre voreltern. 
und scheint noch des weiteren gesichert durch das zweimal vorkommende substantiv orceasnes:

WW 424, 23 inmunitas orceasnes $=491,22$.

Wie im vorigen der ansatz gar keine rücksicht auf das lemma nimmt, so auch im folgenden: $p \bar{a} d$ 'cloak' (OET pg. $592 \mathrm{~b}$ ).

Der Erfurt 1 (C. Gl. L. V 381, 7) bietet

der Epinal hat

praetorsorum paad

der Corpus (P 832)

prętersorim paad,

praetersorium paad und P 670: pretersorim paad.

An einer dritten stelle (P 739) begegnet ein praesorium pund, das sicher hierher gehört und uns den schlüssel zum verständnis der vorgehenden glossen liefert. Wie Loewe im Prodromus pg. 411 richtig gesehen hat, sind praetorsorum und genossen verderbnisse aus pertunsorium (vocabulum vulgare penem significans) und pund ist die einzig richtige lesung an allen stellen $=$ 'Stössel' ', wovon das diminutiv pyntyl veratrum (d. i. veretrum) WW $632,12=$ pyntylle ueretrum WW $636,39=$ pyntyle veretrum, priapus WW 677,30 . 31 vorkommt.

Kluge bringt in seinem etymologischen wörterbuche das deutsche 'Fitze' $=$ 'gebind garn' mit as. fittea, ags. fitt $=$ 'kapitel, gedichtabschnitt' in verbindung. Dieses fitt setzt Sweet (OET pg. 515 a) an auf grund des Erfurt 3 (C. Gl. L. II $566,25)$ ampusatio una lectio fint. Nun bietet aber das fragmentum Deycksianum amputatio unaelectio fĩt, während der Cantabrigiensis hat

\section{amputatio unaelectio siue uineae.}

Daraus ist ersichtlich, dass fint nicht angelsächsisch, sondern abkürzung von sive uitis ist und wir $\mathrm{zu}$ lesen haben amputatio unae lectio siue vitis, das heisst amputatio wird erklärt als 'traubenlese oder weinstock-beschneidung'.

Der ansatz here-searu (OET $483 \mathrm{a}$ ) 'war-stratagem' ist von Sievers schon bei besprechung der Wülkerschen neuausgabe von Wright's Glossen als unsinnig zurückgewiesen worden, da aber Hall in seinem wörterbuch es wieder aufgewärmt hat, so soll nochmals darauf hingewiesen werden, dass des Erfurt 1 (C. Gl. L. V 401, 10 yripeon heresearum und des Corpus (Y 6)

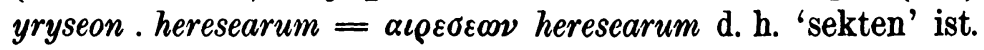

${ }^{1}$ vgl. das Horazische 'alienas permolere uxores' Horat. sat. I, 2, 35. 
$\mathrm{Ob}$ auf grund von Bede ${ }^{2} 54$

\section{et casu and weas}

es geraten ist ein wẽas 'by chance' anzusetzen (OET 611 a) scheint mir mehr als zweifelhaft. Ich sollte meinen, wir haben da einen fall der vertauschung von $u$ und $s$ für $f$ und $r$ vor uns und fear ist nichts als faer, das ja das reguläre wort für casus ist;

cf. WW 200, 40 cassibus calamitatibus uel ferum uel helme d. h. casibus calamitatibus ferum (= faerum) cassibus helmum.

WW 220, 41 cassus inanis scelus malum uacuus uel faer d.h. cassus inanis uacuus, casus scelus malum uel faer.

WW 363, 20 cassus d. $h$. casus faer. ${ }^{1}$

Auch der ansatz wudu-māer 'wood-nymph, echo' (OET pg. $600 \mathrm{~b})$ beruht wohl auf einer voraussetzung, die schwerlich den sinn des glossators trifft.

Augenscheinlich sah Sweet in -maer dasselbe wort, das er pg. $538 \mathrm{~b}$ als mere 'night-mare' anführt. Dass der glossator aber das echo nicht als Wald-mahr erklären wollte, sondern als Wider-märe, d. h. Wider-hall, darauf scheint die überlieferung des Erfurt hinzudeuten, denn da lesen wir (C. Gl. L. $\mathrm{V} 537,20)$

echo uuydumer; das ist, denke ich

uuydirmer und übersetzt jedenfalls ein reverberatio vocis oder etwas derartiges cf. C. Gl. L. V 19, 17

echo graecum nomen est est autem imago uocis quae in concauis locis resultat ostensa ac rosonat (d. h. resonat) ....

OEI pg. $648 \mathrm{~b}$ führt Sweet das verb oefstan 'to hasten' an und unter den belegen dafür erscheint die Corpus Glosse (C 436) circinatio oefsung, aber es kann kein zweifel sein, dass oefsung dasselbe wort ist, das WW 364, 9 als efesung (circinnatio) erscheint und eine ableitung von dem verb efesian ist,

1 Allerdings lesen wir auch WW 401,10 fortuitu mid weas gelimpe, aber auch das könnte mid fear (d. h. faer) gelimpe sein, ebenso Aelfred Boëth. (Corson's Handbok 105, 29) And gif him nu weas gebyrep dürfte sein and gif him nu faer gebyreb $=$ si casus ei accidit. Sievers Gr. $§ 319$ versieht auch wéas mit einem fragezeichen, da er es augenscheinlich für nicht gesiçhẹrt hält. 
dessen Aelfric sich öfters in seiner übersetzung des alten testamentes zur wiedergabe von tondere $\mathrm{u}$. dgl. bedient:

Gen. 41, 14 hine man efosode (totonderunt [eum]);

Lev. 19, 27 ne ge eow ne efesion ne beard ne sciron neque in rotundum attondebitis comam nec radetis barbam;

Deut. 14, 1 ne efesiad eow ne eowre haer ne sciron non vos incidetis nec facietis calvitium;

Lib. Judic. 13, 5 man me mot hine efsian oððe besciran cuius non tanget caput novacula; ibid. 16,17 ic naes naefre geefsod ne naefre bescoren ferrum nunquam ascendit supra caput meum. Sollte Ahd. Gl. II 216, 35 tonsi kiebinota daher gehören und efesian eine ableitung von efen 'eben' sein?

OET pg. $642 \mathrm{~b}$ finden wir auf grund von der Corpus Glosse (A 267) adplaudat onhliorrouuit ein verb rowan angesetzt, ohne dass freilich eine erklärung gegeben wäre. Wenn ich vergleiche Ahd. Gl. I 225, 26 plausus deoh slahanti hantaslagonti, so kann ich die vermutung nicht unterdrücken, dass an unserer stelle on hlior heauuit $\mathrm{zu}$ lesen ist, so dass also das verb heawan hier zu statuieren wäre cf. C. Gl. L. IV 481, 11 alis plantentem (= plaudentem) pennis percutientem.

Ein anscheinend dem deutschen $F$ eim = getreidehaufe entsprechendes ags. fin 'heap' setzt Sweet pg. $507 \mathrm{~b}$ an auf grund von Erfurt 3 (C. Gl. L. II 573, 24 cella lignaria . fin und II 580, 26 lignarium ligneum et est fĩn; hier ist zu bemerken, dass Loewe in seiner ausgabe des Erfurt 3 (Glossae Nominum no. 1083) die worte et est fin liest et est finis, worauf er auch im Prodomus pg. 126 bezug nimmt. Es läge daher nahe, das an erster stelle aufgeführte fin auch $=$ finis zu lesen. Doch gestehe ich gern, dass auch die möglichkeit vorhanden ist, dass Loewe sich betreffs der bedeutung von über dem zweiten fin irrte, denn dieses zeichen erscheint zuweilen auch da, wo von einer abkürzung nicht die rede sein kann.

OET' pg. $471 \mathrm{~b}$ finden wir den ansatz slagu 'slag' d. h. schlacke auf grund von Erfurt 1

(C. Gl. L. V 369, 47) lithargum slaegu,

Epinal ibid. lithargum slegu,

Corpus (L 247) lihargum slaegu.

Augenscheinlich hat Sw. in lithargum eine verderbnis von lithargyrum $=\lambda \iota \vartheta \alpha \rho \gamma v \rho o v$ gesehen und darnach seinen ansatz gemacht. Aber wir haben es doch wohl hier mit lithargum = 


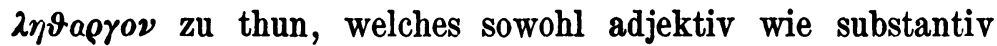
sein kann. Ich vermute, es ist hier substantiv und slaegu ist = slaege 'schlag', 'plötzliche krankheit' vgl.

C. Gl. L. IV 106, 27 letargus uitium quo compremuntur egri ad falsum somnum.

IV 107, 25 letagito (?) infirmitas somnium infirmum somnium,

IV 109, 32 litargicus sommulentus,

IV 254, 38 leargus uitium quo compremuntur egri ad falsum somnum,

IV 255, 35 litargus somnulentus,

V 307, 18 letargus uitium quo comprimuntur egri.

IV 533, 24 laetragus (= laetargus) uitium quo compraemuntur aegriae aut (= aegri ad) somnum.

OET pg. 576 a haben wir den ansatz horweg aj. 'muddy' und dafür wird als beleg angeführt aus dem Erfurt1 (C. Gl. L. V 356, 47)

und aus dem Corpus (D 72)

deuia callis horu aegstug

deuia callus horweg stig.

Es sollte doch auf den ersten blick klar sein, dass horuaeg stiig, horweg stig nichts anders ist als orweg stiig d. h. 'wegeloser (unwegsamer) steig (pfad)', aber Hall hat getreulich die Sweet'sche erfindung kopiert.

OET 504 a gewinnt Sweet ein wort clifeht 'cleaving' aus folgenden glossen:

Erfurt 1 (C. Gl. L. V 349, 23) cliborum clibectis; Epinal (ibid.) clibosum clibecti; Corpus (C 443) clibosum clibecti.

Wie er dazu kam, ist kaum verständlich. clifeht ist natürlich 'kliffig', 'schroff', 'steil', wie WW III 36 cliuosus clifig tohyld deutlich beweist, doch Hall hat getreulich wieder kopiert.

Der ansatz (OET pg. 646a) poden 'whirlwind' auf grund von Corpus (A 482) alcanus (d. h. altanus) poden scheint durchaus gerechtfertigt, da das wort auch anderwärtig bezeugt ist. Wir treffen es wieder

WW 109, 7 altanus poden

WW 203, 5 ceruleis turbinibus lageflodum podenum.

Two Saxon Chronicles a. 793 (D) her waeron reðe forebecna 
cumene ofer Norðanhymbra land 7 $\not$ folc earmlice bregdon; $\not$ waeron ormete podenas 7 ligrescas.

Byrhtferth's Handboc (128) paet manega men geseoð feallan of paere heofone swylce hyt syn steorran. hyt beod spearcan (129) of pam rodere purh paes windes blaes pe paenne swydlice ba heannyssa paes roderes scecð mid his podenum.

Ich kann mich indes des gedankens nicht erwehren, dass wir an allen stellen woden herzustellen haben, da ich glaube, dass überall die rabies venti ausgedrückt werden soll und sicherlich kann ein verheerender sturm nicht passender bezeichnet werden, als mit dem namen Wodans von dem man ja noch heute im volke glaubt, dass er mit seinem wilden heere durch den wald ziehe, wenn die frühlingsstürme brausen. Wegen der form woden vgl. WW 245, 10 furor enim animi cito finitur uel grauius est quam ira repnes woden dream. Ueber die vertauschung von $w$ und $p$ vgl. Corpus C 397 cicuta wodewistle d. h. wodepistle.

OET pg. 485 a setzt Sweet ein mearh 'horse' an auf grund von Corpus (A 536) amilarius mearh; aber ein solcher ansatz ist nur möglich, wenn man das lateinische lemma ganz und gar missachtet. Wollen wir jedoch der meinung des glossators auf die spur kommen, so müssen wir gerade auf amilarius zurückgehen. Das wort sieht aus wie eine ableitung von

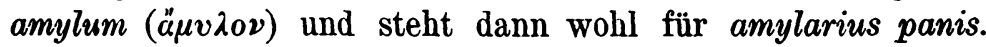
Vielleicht ist es eine nachbildung von áuv́zıoc, das Exod. 16, 31 begegnet (Aq.) Ich vermute, die glosse hat ursprünglich ungefähr so gelautet:

amylarius ab amylo dictus id est medulla frumenti 1 .

Wegen mearh vgl. WW 159, 32 medulla uel lucanica mearh.

Keinerlei spur von dem bei WW 42, 19 als ags. bezeichneten fahame habe ich bei Sweet entdecken können. Die überlieferung ist die folgende:

Erfurt 1 (C. Gl. L. V 381, 9):

pulmentum fahamae;

Epinal (ibid.):

pullentum fahamae;

1 vgl. Ahd. Gl. I 367, 1 medulatrici (= medulla tritici) io simila = Deuteron. 32, 14. 
Corpus (P 562):

Corpus (P 874):

polentum fahame;

pullentum fahame.

Wenn wir vergleichen Corpus C 497 polenta smeodoma (ibid. S 345 simila . farina . subtilis) und uns erinnern, dass dieses eine vom particip des verbs smeagean mit dem suffix -ma gebildetes wort ist und also ursprünglich 'prüfung, reinigung' bedeutet haben muss, so läge nahe, eine ähnliche bildung für fahame anzunehmen, nämlich fahame, wo dann -me für -ma stehen müsste, aber das -mae des Epinal-Erfurt macht mich stutzig und ich vermute, das mae ist verstümmelung von mael, so dass wir also fahamael (fahamel) zu lesen hätten, worunter ich 'fein (gesiebtes) mehl verstehe, vgl. ahd. [fawjan, fowjan] fowen, mhd. vaewen = bair . feben, fehen cribrare (Schade, Ahd. Wtb. I 172b).

Bei dieser gelegenheit will ich auf das im Corpus C 910 vorkommende

\section{crebro pugillo}

aufmerksam machen, das im Erfurt 2 (C. Gl. L. V. 282, 13)

crebro pugillo

wieder begegnet und mit dem Corpus P 514 erwähnten pocillus genus panis ${ }^{1}$ im zusammenhang $\mathrm{zu}$ stehen scheint.

Das obige crebro dürfte wohl = cribro sein, und steht pugillo (mit austausch von $g$ für $c$ wie so oft) für pucillo (pocillo), dann ist cribro dativ von cribrum 'sieb, gesiebtes, aus gesiebtem mehle gemachtès' d.h. pocillus pucillus müsste eine bildung vom stamme pu- 'rein' sein, der in purus vorliegt; wir hätten also ein lateinisches wort, das einiges licht auf obiges ags. fahame(l?) werfen dürfte.

OET pg. $466 \mathrm{~b}$ erschliesst Sweet ein

$$
\text { holo-panne 'pan' }
$$

aus Epinal (C. Gl. L. V 381, 16):

und Corpus P (73):

$$
\text { patena holopannae }
$$

$$
\text { paneta holoponne. }
$$

1 Erscheint auch im Erfurt1 (C. Gl. L. V 352, 3) und im Epinal (ibid.). Es ist freilich möglich, dass pugillus Levit. 2, 2 gemeint ist (pugillum similae 'eine handvoll mehl') und die erklärung genus panis ein missverständnis der stelle ist. In dem falle ist crebro pugillo schwer zu erklären, pugillo müsste denn verb sein und für purgillo (?) reinigen (?) stehen. 
Was es aber mit diesem holo- für eine bewandtnis hat, darüber erhalten wir keinen aufschluss. Ich glaube, wir haben hier zwei zu trennende wörter vor uns: holo (verstümmelt aus heolor) und panne, d. h. der glossator wollte sagen patena kann eine 'wagschale' und eine 'pfanne' bedeuten. Vgl. C. Gl. L. V 305, 46 lance patellice id mensura,

306, 47 lancibus pandis (d. h. panetis = patenis (siehe oben)), catenis (d. h. catinis) uel alubiolis (d. h. alviolis). !

So haben wir Corpus L 16 laxheholor d. h. lãx $=\operatorname{lan} x$ heholor $=$ heolor wie aus des Epinal (C. Gl. L. V 368, 37)

$$
\text { laxhe olor }=\text { lanx heolor }
$$

deutlich hervorgeht. Der Erfurt 1 hat das $h$ verloren (C. Gl. L. V 368, 37)

aber V 369, 53 hat er es:

$$
\text { laxe olor, }
$$

\section{lanx helor.}

Was Sweet OET pg. $505 \mathrm{~b}$ aus Corpus 24 gewinnt, nämlich gerinen ptc. 'diligent' ist zwar schon von Hessels in seiner ausgabe des Corpusglossars zurückgewiesen worden. Da aber Hall es trotzdem gläubig aufgenommen hat, so dürfte es angebracht sein, an dieser stelle nochmals darauf hinzuweisen, dass Hessels zu Introd. 229 nauum germen bemerkt, der name (jedenfalls ist es der des propheten Nahum) finde sich mit der erklärung in des Hieronymus' Liber de nominibus Hebraicis.

Auch der 'fisch', den Sweet in Corpus $66=$ Corpus A 160 acus netl $\ell$ gronuîsc sah, ist von Dieter letzthin als 'Granne' erwiesen worden. Ich erwähne es hier nur, weil es charakteristisch für die art ist, mit der Sweet bei aufstellung seiner wörter verfahren ist: das lateinische Lemma existiert für ihn einfach nicht, wenn es nicht im einklange mit seiner annahme eines angelsächsischen wortes steht.

So nimmt es denn auch kein wunder, wenn er OET p. 495a ein wort leactrog 'lettuce' auf grund von Epinal 247 leactrocas corymbus aufstellt. Z Zwar wissen wir von keiner stelle, wo corymbus 'salat' bedeuten könnte, aber das nach lactuca modellierte angelsächsische leactuc erscheint mehremals in der verderbten form leahtric (so WW 297, 18. 432, 7) und das genügte für Sweet, hier an unserer stelle dem corymbus ein

1 vgl. auch Glossae Nom. 1059 lancx patina tali figura.

Anglia. N. F. VII. 
leactroc = leactric 'lettuce' unterzuschieben und so finden wir denn auch bei seinem getreuen abschreiber Hall die einträge leactrog -troc -tric = leah-troc -tric -trog 'lettuce'.

Sehen wir uns die handschriftliche überlieferung ein wenig näher an. Der Epinal hat (Corp. Gl. L. V 253, 27) ebenso wie der Erfurt corimbus leactrocas; der Corpus (C 656) corimbos leactrogas. Es ist klar, dass auf grund des Corpus auch im Epinal-Erfurt corimbos für corimbus zu lesen ist. Wir haben demnach augenscheinlich ein leactroc $=$ corymbus zu statuieren. Was aber bedeutet das? Sehen wir uns nach andern stellen um, so finden wir WW 213, 16-19

corimbi i. uiti racemi uel botriones uel circuli wingeard hringas uel bergan uel croppas bacce.

corimbus cacumen nauis leahtroh.

Nach zuletzt angeführter stelle müsste die bedeutung von leactroc '(leahtroh) etwa 'schiffsspitze, schiffsschnabel' sein; man sollte aber dann doch eher ein wort wie heahðu erwarten, und es ist möglich, dass dies wort in leahtroh steckt; das anlautende $h$ kann nach beliebter manier ausgelassen worden sein und $l$ für $\ell$ stehen, so dass zu lesen wäre $\ell[h]$ eahthu $=$ $\downarrow$ heahðu. Aber es ist ebenso gut auch möglich, dass leahtroh gar nicht interpretament zu corimbus cacumen nauis ist, sondern zu dem vorhergehenden gehört, was man mit um so grösserer wahrscheinlichkeit annehmen könnte, als die ursprüngliche ordnung dort sicher gestört ist. Denn es ist klar, dass bergan $\mathrm{zu}$ dem später folgenden bacce gehört wie auch croppas nicht an seiner gehörigen stelle steht. Vielleicht ist zu ordnen

corimbi $i$. uitis racemi cnoppas uel botriones leachocgas uel circuli wingeard-hringas uel bacce bergan.

leachocgas möchte sich empfehlen auf grund von WW 201, 31 caprioli dicti quod capiant arbores wingeard-hocgas; WW 118,3 capreoli wingeardbogas $=w$. g. hogas und so wäre denn statt des Sweet'schen leactroc 'lettuce' ein leachoc 'lauchhaken', ranke anzusetzen. Doch ist das durchaus nicht sicher. Jedenfalls aber kann von einem leahtrog = lettuce keine rede sein, Kolkwitz hätte das beweismaterial sorgfältiger prüfen sollen, ehe er Anglia XVII 4, 459 Sweet's aufstellung zu seiner eigenen machte. Was er da von einschiebung eines $r$ sagt, gilt lediglich für leahtric WW 297, 18. 432, 7. 
OET pg. 566 a folgert Sweet ein byrde aj. 'of high rank' aus Erf. 1153 byrdistrae blaciarius primicularius. Diese bedeutung hat Sw. jedenfalls aus dem interpretament primicularius gewonnen. Aber wie schon Loewe zu Glossae Nominum 312 (= Erf. 1153) vermutete, ist dafür primicu[bicu]larius zu lesen und dies ist, wie ich im American Journal of Philology ausgeführt habe, interpretament $\mathrm{zu}$ einem ausgefallenen lemma blasto, dem namen des Apostelgesch. 12, 20 erwähnten Blastus, qui erat super cubiculum regis; d. h. wir haben zwei glossen zu einer verschmolzen:

a) Blasto primicubicularius,

b) blatiarius (so liest Loewe) byrdistrae saconice.

Was aber ein blatiarius ist, lernen wir aus dem Cyrillus-

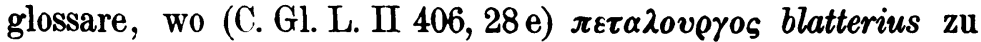
lesen ist, d. h. blattearius steht für bractearius; $\pi \varepsilon \dot{\tau} \tau \lambda$ גov aber ist ein streifen, eine platte cf. Corpus Gl. P 240 petalum . laminea aurea . in fronte in qua scriptum nomen dei. tetragrammaton C. Gl. L. II 406, 27 a bratteum (= bracteum) flaminium (= flaminun) lamina, bezieht sich also augenscheinlich auf das brustschild der jüdischen priester. Wenn wir nun erwägen, dass WW 148, 12 wir lesen brattea (= bractea) gylden laefr d. h. doch wohl 'goldene "Läuber" (wenn dieser plural gestattet ist) $=$ goldene blätter; besonders aber

WW 360, 33 bratea (= bractea) gylden pel arlaegen (?),

$\mathrm{WW} 358,15$ bratheas (= bracteas) goldfel = goldpel,

WW 518,4 petala goldfyld fel = gold pylo pel,

so wird wahrscheinlich, dass byrd in byrdistre eine nebenform zu dem gewöhnlichen bred = brett ist, das 50, 1;347, 42; 528,$33 ; 499,40$ und in der schreibung bret 107, 6 und 485, 5 vorliegt, wo für writbrec zu schreiben ist writbret. Wenn aber der glossator die masculinform bractearius durch die femininform byrdistrae $==$ 'brettlerin' wiedergab; so wollte er jedenfalls andeuten, dass die verfertigung eines solchen schmuckgegenstandes bei seinem volke frauenarbeit sei.

Da wir einmal bei dem stamm byrd- stehen, so mögen noch einige andere worte hier erörtert werden, die anscheinend dazu gehören.

OET 477 a führt Sweet ein substantiv wynde-craeft 'art of embroidery' an. Dasselbe fusst auf Cp. 217 uuyndecraeft ars plumaria und Rd. 9 *uyrdicraeftum. Nun finden wir WW 
294, 10 ein plumaria byrdinge und augenscheinlich dasselbe ist, was WW 262, 18 als plumaria byrdicge erscheint. Sodann WW 205, 21 haben wir clauatum sutum uel gebyrd, WW 375, 41 clauatae bebyrde oððe bestefnde. Es ist erklärlich, dass Rd 9 zusammengehalten mit WW 294, 10, an beiden stellen dasselbe wort vorzuliegen scheint. Es handelt sich also darum, sollen wir mit Sweet in der überlieferung des Rätsels ein versehen für wyndicraeftum annehmen, oder darin nach analogie von byrdingc eine andere schreibung von byrdicraeftum sehen? Die entscheidung wird uns nicht schwer fallen, wenn wir erwägen, dass C. Gl. L. V 525, $3=525,16$ plumarium durch clauatum erklärt wird, was wir oben durch gebyrd oder bebyrd wiedergegeben sahen. Die ars plumaria ist demnach eine byrdicraeft d. h. 'stick-kunst' (cf. Corpus C 212 opere plumario bisiudi werci) und byrd-ist der um ein $r$ erleichterte stamm bryrd der WW 421,5 in onbryrde (instigauit), WW 419, 42 onbryrdinge (instinctu) erscheint und umlaut von brord ist, dass wir WW 277, 12. $470,16=$ punctus haben (cf. Ahd. Gl. II 706, 52 picto limbo gebrorderemo soume. Diese um ein $r$ erleichterte form ist noch heute im Niederdeutschen gang und gäbe, cf. 'Dat Brüden geit um' = Das Sticheln geht um, d. h. neckst du mich, so neck ich dich, wie du mir, so ich dir.

Mit diesem byrd ist nicht $\mathrm{zu}$ verwechseln ${ }^{1}$ was $=$ beard in WW 420, 7 pa ungebyrdan heapas (inuestes d. h. bartlos, jung cateruas) WW 425, 31 inuestis ungebyrd und in frumbyrdling d. h. erstbärtling = flaumbart, milchbart WW 171, 23 (pubetenus) erscheint. Hall (Concise Anglo-Saxon Dictionary) konnte aus dem letztern nicht klug werden und so nahm er einfach die ganze glosse in sein wörterbuch auf, aber es ist klar, dass pubetenus versehen für pubes iuvenis (oder möglicherweise auch für pubescens) ist.

1 Vielleicht besteht aber doch ein innerer zusammenhang zwischen byrd (= bryrd) 'stachel, spitze, stich' und byrd (= beard) 'bart'. Es ist gewiss nicht zufällig, dass man die grannen der ähren 'bart' im Deutschen wie im Englischen nennt, oder die grasspitzen, das aufkeimende getreide breird im Schottischen. Ist es nicht denkbar, dass beide wörter auf e in er vorstellung beruhen? Jedenfalls ist Kluge's zusammenstellung von Bart mit barba durchaus nicht befriedigend.

Hartford Ct. U. s. A., März 1896.

Otтo B. SchuUtter. 